\title{
A Query Language for Data Access in Ubiquitous Environments
}

\author{
Cristiano Silveira \\ Mestrado Integrado Profissional em Computação \\ Universidade Estadual do Ceará (UECE), Ceará, Brasil \\ cristiano.silveira@uece.br \\ Leonardo Eloy \\ Centro de Ciências Tecnológicas \\ Universidade de Fortaleza (UNIFOR), Ceará, Brasil \\ eloy@unifor.edu.br \\ José Maria Monteiro \\ Departamento de Computação \\ Universidade Federal do Ceará (UFC), Ceará, Brasil \\ monteiro@lia.ufc.br
}

\begin{abstract}
Recent progress in ubiquitous computing technology is allowing development of new and sophisticated applications that are now able to store very large data on the mobile device itself, besides access data stored remotely. Thus emerges the need to query and maintain data from portable equipments. However, traditional query languages such as SQL (Structure Query Language) and QbE (Query by Example) are inadequate for devices with small screens. Hence, this work presents QbZ (Query by Zoom), a query language based on Semantic Zoom for small-screen devices. The proposed mechanism is particularly suitable to locate and access data stored in portable equipments. In order to demonstrate the benefits of using QbZ, usability tests were conducted, as well as a comparative analysis with the existing solutions.
\end{abstract}

Keywords: Query Language, Ubiquitous Environments, Semantic Zoom

\section{INTRODUCTION}

Recent progress in technology used in portable devices (such as PDAs, cell phones and smartphones) is contributing to diminish the cost of these equipments, making them accessible to a growing group of persons. This progress has contributed to increasing the computational and storage capacities of portable equipment. In the beginning of 2008 , HTC, a smatphones manufacturer, introduced the HTC X9501, which has a 40 GB HD [11].

Today, with the advancement of technology in both data communication networks and accessing devices, new and sophisticated ubiquitous applications can be developed, the demand for which should follow the growth trend. Examples of these applications are: teaching and collaborative working, electronic tourist guides, navigation and route planning [1]. Thus, these new applications will handle and store a large volume of information. Consequently, emerges the need to query available data. However, traditional query languages, such as SQL (Structure Query Language) and QbE (Query by Example), are inadequate for devices with small screens [12]. Thereby, it is very important to investigate new paradigms and metaphors to design query languages toward ubiquitous environments. 
This work introduces QbZ (Query by Zoom), a query language based on Semantic Zoom [13] for smallscreen devices. The proposed mechanism makes it easier to locate and access data.

This article is organized as follows: Section 2 presents the concepts necessary to understanding the proposed language; Section 3 discusses related works; Section 4 introduces the QbZ language; Section 5 presents the results of tests undertaken; Section 6 concludes this work and points out directions for future works.

\section{BASIC CONCEPTS}

The success of a database system is, to a great extent, connected to the ease of recovering stored information. It is through the database query language that the user is able to express a set of restrictions and select the information desired.

A query language is defined as a high level computing language for recovering and modifying data. A relational query language can be understood as a formally well defined set of operators that can be combined to express queries in databases [5]. The process of formulating the query is carried out in three steps [8]:

1. Location: the user selects a set of information of his interest, that is, objects that will make up part of his response set, or that will be used to restrict the set of values of the response;

2. Definition of requirements: some restrictions are applied to the objects selected in the previous phase, and these restrictions must be satisfied in order for such objects to become a part of the query result;

3. Viewing the result: the result of the query is presented to the user. The same set of information may be presented in different ways, but the choice of the adequate representation allows better interpretation of the results by the user.

Query languages can be classified into two large groups: text languages and visual languages. A text query language (SQL, for example) requires the user to have great knowledge of syntax and of the manner in which the information is stored in the database. Visual query languages, or Visual Query Systems (VQS), present a friendlier environment for recovering information, allowing users unfamiliar with text language syntax to formulate their queries in a simple and intuitive manner [8].

The Visual Query Systems (VQS) use a language to express the queries in visual format, and a variety of functionalities to favor interaction of the user with the system [8]. VQS can be seen as an extension of DBMS (Database Management Systems), allowing data queries to be formulated by less experienced users.

A VQS can be divided into two parts: external query model, and internal query model [5]. The external model defines the manner in which the user will visualize and manipulate the information. The internal model, in turn, is used to represent the queries elaborated by the user through the external model, in the query language defined by the implementation environment. For example, the external query model may use QbE, while the internal model uses SQL. In this case the user elaborates his query using QbE, and this query is mapped to SQL, so it can be executed on the DBMS. In order to allow translation between the external and internal models, a mapping module must exist that promotes the relationship between the operations and representations of each model [5].

The external model must present great power of expression and a very friendly query language. The internal model on the other hand, depends on the DBMS used and the query language it supports. The possibility of using different models allows the environment for interaction with the user to be defined without considering the implementation aspects. 
VQS can be classified into four paradigms: form-based language, diagram-based language, iconbased language, and hybrid language [5]. Although VQSs have been used with considerable success in applications that execute in personal desktop computers, their use in small-screen devices, like cell phones and smartphones, for example, has not proved adequate [12]. Hence, the investigation and definition of new paradigms for the construction of VQSs that are more appropriate for portable devices have become an important research area.

\section{RELATED WORK}

Massari [12] presents a prototype of a visual query system that supports the database exploration and query from mobile devices based on the icons manipulation. This prototype is called Query By Icons (QBI). In this approach the user primarily interacts with the system with a pointing device, such as a pen or a mouse, and composes query by arranging icons. However, this prototype was not implemented for mobile phones.

In [7] the authors propose a graphical query language called MoSQL to be the basis of general mobile information systems. This language has an icon-based interface. That is, the same class of data represented in the database system corresponds to the same icon. This windows-based interface is suitable for mobile devices that can be operated by clicking or dragging the mouse, like notebooks and PDAs. So, it is not suitable for mobile phones.

The paper [2] presents the design of a high-level web-based user interface using IRE (Information Requirement Elicitation), in the context of "Hospital database queries" by a mobile web user. The prototype is based on the notion of Query-By-Object (QBO) approach of building a query using multiple user-level steps. The Query-by-object (QBO) interface has been studied for Geographic Data Systems.

In [1] the authors introduce a free-form database query language which was implemented in a database query system prototype for WAP-enabled mobile phones. Free-form is a concept which is based on the universal relation [1]. It can be used to reduce the number of terms needed in a query. Hence, using the language, any relational query can be formulated without the need to provide precise inputs. However, the imprecise input may lead to many interpretations in terms of the attributes to be displayed as outputs. Furthermore, the prototype makes intensive use of menus and the database schema is shown in a very limited manner (using in a list component).

Polyviou, et al., [14] discussed expressive queries in their work, denoted Query by Browsing (QBB), which implements a directory-like interface for query formulation. However, their method is suitable only to be used with devices that have pen input mechanism. As the majority of mobile phones lack this type of input mechanism, we believe that a conventional way of input, i.e., input using normal phones keypad and function keys, should be considered. Another interesting option to be considered for inputs would be voice input. However, as being highlighted in [6], this method has one major problem of voice recognition which needs further research.

In this paper we present QbZ (Query by Zoom), a query language based on Semantic Zoom, for portable devices. QbZ is a hybrid language and uses aspects from diagram and icon paradigms. The "Browsing" technique is used to understanding the reality of interest and the "Schema Navigation" to query formulation. QbZ can be used with different input devices like Pointing devices (pen and mouse), phones keypad and functions keys. The QbZ interface is operated by menus, lists and arranging icons. Moreover, QbZ has some additional features, such as: visual representation, definition of detail levels, navigation on the graph scheme. Figure 1 shows a comparative analysis between QbZ and previous languages. 


\begin{tabular}{|c|c|c|c|c|}
\hline VQS & Paradigm & Conception & Input Devices & Interface \\
\hline QBI & Icon & $\begin{array}{l}\text { Not for mobile phones. } \\
\text { But, can be adapted } \\
\text { for mobile devices }\end{array}$ & $\begin{array}{l}\text { Pointing devices } \\
\text { (pen or mouse) }\end{array}$ & $\begin{array}{l}\text { Operated by arranging } \\
\text { icons }\end{array}$ \\
\hline MoSQL & Icon & Not for mobile phones & Mouse & $\begin{array}{l}\text { Windows-based. Op- } \\
\text { erated by clicking or } \\
\text { dragging the mouse }\end{array}$ \\
\hline QBO & Hybrid & Not for mobile phones & Mouse & $\begin{array}{l}\text { Web-based. Operated } \\
\text { by clicking or dragging } \\
\text { the mouse }\end{array}$ \\
\hline Free-form & Hybrid & For mobile phones & $\begin{array}{l}\text { Pointing devices } \\
\text { (pen or mouse) }\end{array}$ & $\begin{array}{l}\text { Web-based. Operated } \\
\text { by menus and lists }\end{array}$ \\
\hline QBB & Hybrid & $\begin{array}{l}\text { For desktops and } \\
\text { handhelds }\end{array}$ & Mouse & $\begin{array}{l}\text { Windows-based. Op- } \\
\text { erated by clicking or } \\
\text { dragging the mouse }\end{array}$ \\
\hline QbZ & Hybrid & For mobile phones & $\begin{array}{l}\text { Pointing devices } \\
\text { (pen or mouse), } \\
\text { phones keypad and } \\
\text { functions keys }\end{array}$ & $\begin{array}{l}\text { Based on Semantic } \\
\text { Zoom. } \\
\text { by menus, lists and } \\
\text { arranging icons }\end{array}$ \\
\hline
\end{tabular}

Figure 1: Comparative Analysis

\section{Query By Zoom Language}

The objective of this section is to present and describe the QbZ (Query by Zoom) language. QbZ consists of a visual query language based on Semantic Zoom [13], directed to small-screen devices. The QbZ language follows the standard organization of visual languages, containing an external model and an internal model. The external model uses Semantic Zoom as main metaphor, while the internal model uses the SQL text language.

The basic idea of Semantic Zoom is to allow the user to have control over a large volume of visual objects [4]. As the user closes in on the information, more details of said information are exhibited [13]. Control is carried out by using: Zoom In, whereby the user closes in on the desired information and, depending on the approximation, there is a level crossing; Zoom Out, whereby the user backs off from the information; and Pan, where there is a lateral movement at the same level. Although it is not a recent idea, interfaces based on Zoom have become more prominent in the construction of visual interfaces for portable devices [4].

\subsection{The External Model}

A query in QbZ is represented by a sequence of actions. These actions can be classified in three categories:

- Visual actions of semantic zoom;

- Query formulation actions;

- Data recovery actions;

In all, QbZ offers 15 operations (or actions), as shown in Figure 2: 


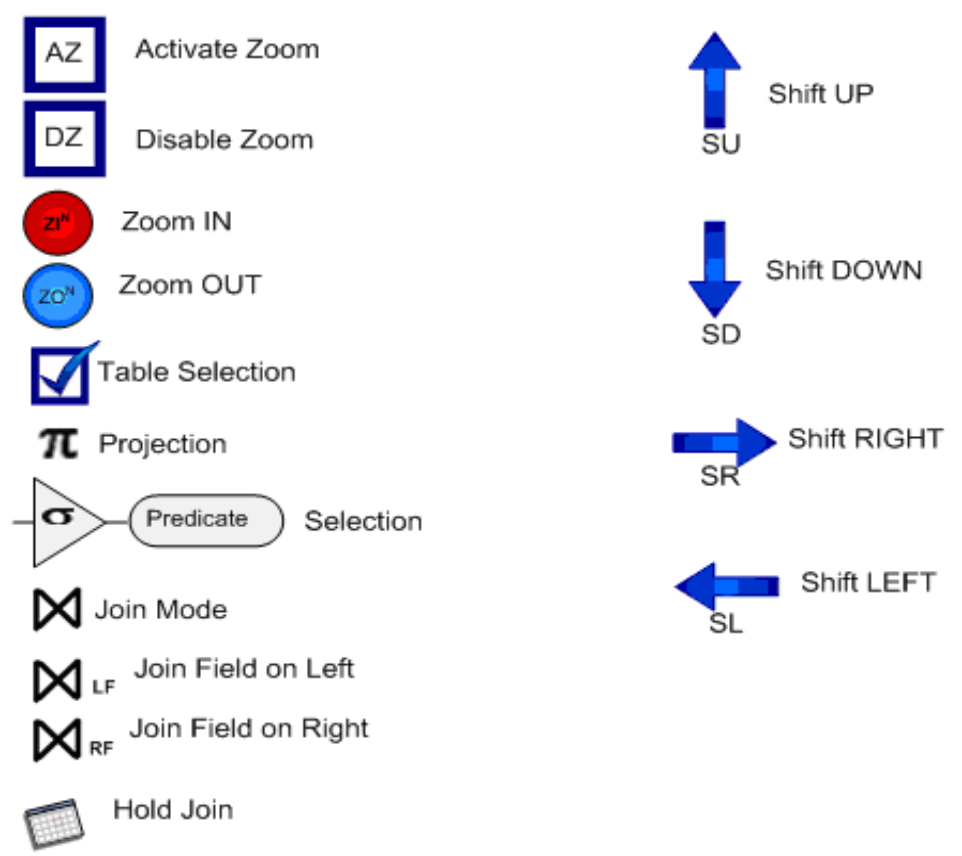

Figure 2: Operators of the QbZ External Model.

- AZ: Activates ZOOM mode. From this point onward the keys of the mobile device are ready to activate the ZOOM IN and ZOOM OUT modes.

- $Z I^{N}$ : Indicates ZOOM IN. Enlarges the selected screen by "n" times.

- $Z O^{N}$ : Indicates ZOOM OUT. Reduces the selected screen by "n" times.

- DZ: Disables ZOOM mode.

- SHIFT UP, SHIFT DOWN, SHIFT LEFT, SHIFT RIGHT: Activates displacement, displacing the visualization content, changing the part that will become visible on the portable device screen.

- TABLE SELECTION: Selects a table.

- PROJECTION: Selects columns in the indicated table.

- SELECTION: Selects the lines of the indicated table that satisfy a certain condition (predicate).

- JOIN MODE: Indicates the start of a joining operation.

- JOIN FIELD ON LEFT and JOIN FIELD ON RIGHT: Indicate the columns that will make up the joining condition.

- HOLD JOIN: Concludes a joining action.

\subsection{The Internal Model}

The internal model of the QbZ language uses the SQL text language. Thus, the queries elaborated using the external model of QbZ must be translated to SQL. 


\subsection{Translation from the External Model to the Internal Model}

A query elaborated from the external model of QbZ corresponds to a sequence of actions. This sequence of actions can be represented by a graph. In order to make it easier to understand the process of translating from the external model to the internal model, we will represent each action that composes the external model of the QbZ with a symbol. Figure 2 illustrates the actions that compose the external model of the QbZ language.

The translation process starts by capturing the actions executed by the user by means of the external model [5]. Next, a graph of QbZ operators is generated to represent the query elaborated by the user. The next step consists of mapping the QbZ operator graph to a graph of relational algebra operators. Note that, for this, the visual actions (operations) of Semantic Zoom are discarded, as are the data recovery actions that may exist on the QbZ operator graph. To conclude the translation process the relational algebra operator graph is translated to a text query in SQL.

\subsection{Semantic Zoom Levels}

The designed strategy uses three levels of Semantic Zoom: the level 1, which initially shows information about the database schema; the level 2, appears after selecting a table and shows information about the selected table, such as: name, size and type of attributes, etc; and the level 3, displayed after the selection of a particular attribute, which displays the details related to the selected attribute and the actions that can be executed from this attribute, as the addition of a projection or a selection condition. These three levels are exemplified in Figure 3.

\subsection{Strategies for User Interface Design}

According to [5], the goal of the people working with a VQS is to retrieve the desired data. This is usually accomplished through the following two main activities:

1. Understanding the reality of interest. The goal of this activity is the precise definition of the fragment of schema involved in the query. Generally, the schema is much richer than the subset of concepts which are involved in the query. The first step corresponds to the identification of concepts that are useful for the query, and the result is a query subschema, i.e. the static representation of such concepts.

2. Formulating the query. The query subschema can be manipulated in several ways, according to the available query operators. The goal of query formulation is to formally express the operands involved in the query, with their related operators.

Understanding the reality of interest by the user is a task that previously was not considered by the query systems. The complexity of this task is directly related to the size of the database schema and workload. This process of understanding the model can be very slow, depending on the knowledge that the user already has from the model. The developed strategies try to ensure a gradual construction of the mental model, with the information already caught about the analyzed data model. All new information captured generate a change in the mental model trying to make it close to reality [5]. There are three strategies that can be adopted in order to facilitate this task: Top-down, Browsing and Schema Simplification [5].

- Top-down. In a top-down strategy, the general aspects of the reality are first perceived, and then the specific details may be viewed. The top-down strategy is implemented in several ways. The first one can be seen as a sequence of iterative refinements, i.e. the system provides for each schema a library of top-down refinements. Each refinement can be obtained from the previous one by means of transformations, which when applied to atomic objects, result in more detailed structures. 


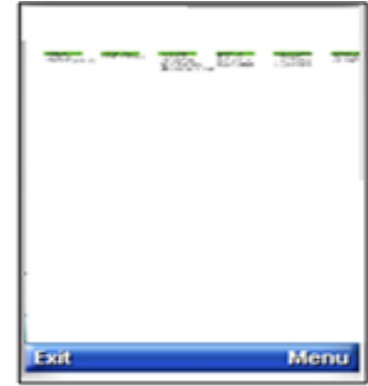

The application starts at the first level showwing the database schema

Level 1 - View 1

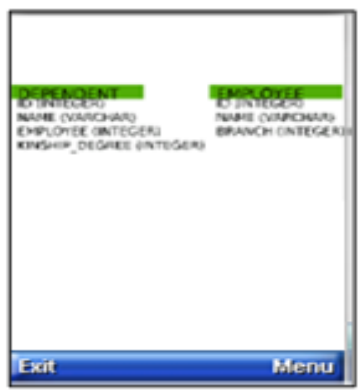

The user runs Zoom in,

closing to the tables Dependent and Employee

Level 1 - View 3

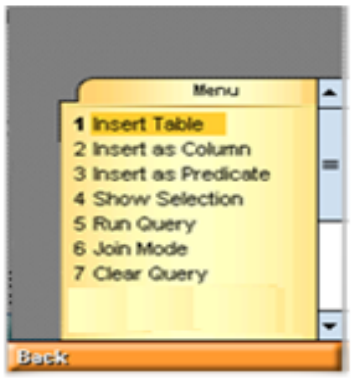

The user selects the Dependent table and it is included in the query. The level of showed details is increased.

Level 2 - View 5

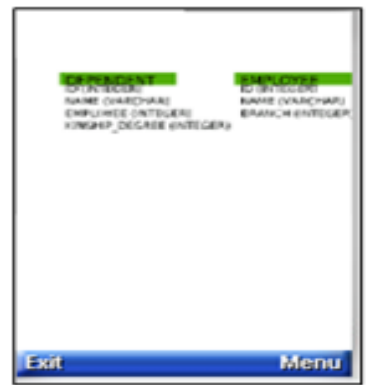

The user turns the Zoom and runs Zoom In, closing to na area of interest.

Level 1 - View 2

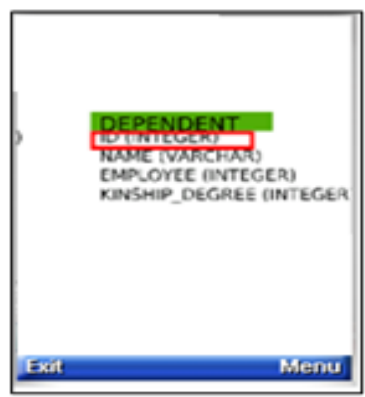

The selection area of the table Dependent is turn on. In this moment, the user can select the Dependent table and goes tothe second semantic level

\section{Level 2 - View 4}

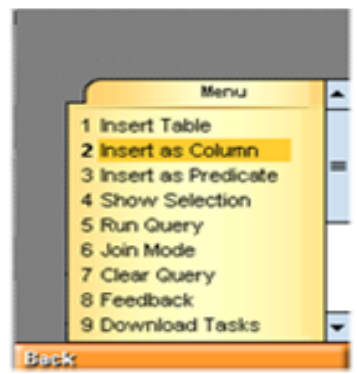

The column selection on area is turn on. In this moment, the user can select a column and goes to the third semantic

level

Figure 3: Semantic Zoom Levels. 
- Browsing. This technique consists in the study of element by element in a diagram of entities. Each selected element is checked, the useful information is acquired by the user and the process continues on neighboring elements. This navigation can be done on the database intention, database extension, or on the database scheme.

- Schema Simplification. The idea here is to "bring the schema close to the query". This is done by building a user view resulting from aggregations and transformations of concepts of the original schema. While in the top-down approach it is possible to locate concepts that exactly match the initial schema (at different levels of abstraction), in the schema simplification approach the user may build a proper view of the original schema which cannot be extracted by the schema itself at any of its levels of abstraction.

Query formulation is the fundamental activity in the process of data retrieval. For this task, the main techniques that can be used are: Schema Navigation, Sub-queries and Matching [5]. The query formulation strategy by schema navigation has the characteristic of concentrating on a concept (or a group of concepts) and moving from it in order to reach other concepts of interest, on which further conditions may be specified. Such a strategy differs according to the type of path followed during the navigation [5].

In the mechanism proposed in this paper we used the technique "Browsing" to understanding the reality of interest. For the query formulation task was used "Schema Navigation". However, to implement browsing and schema navigation we propose to use Semantic Zoom.

In the case of Semantic Zoom, the schema is unique, and the concepts are layered in terms of levels of importance; the schema can be examined at several levels, so that only objects above a specified importance level are visible. The user can also graphically edit the schema, so that irrelevant objects can be removed from the screen. The schema is also unique, but the objects may be examined at different hierarchical detail levels. Next, we present more details about the Semantic Zoom technique.

\section{Zoomable User Interface:}

A zooming user interface or zoomable user interface (ZUI) is a graphical environment where users can change the scale of the viewed area in order to see more detail or less [4]. A ZUI is a type of graphical user interface (GUI). Information elements appear directly on an infinite virtual desktop (usually created using vector graphics), instead of in windows. Users can pan across the virtual surface in two dimensions and zoom into objects of interest. For example, as you zoom into a text object it may be represented as a small dot, then a thumbnail of a page of text, then a full-sized page and finally a magnified view of the page [9].

Perlin suggests: Imagine that the computer screen is a section of wall about the size of a typical bulletin board or whiteboard. Any area of this surface can then be accessed comfortably without leaving one's chair. Imagine further that by applying extraordinarily good eyesight and eye-hand coordination, a user can both read and write as comfortably on any micron wide section of this surface as on any larger section. This would allow the full use of a surface which is several million pixels long and high, on which one can comfortably create, move, read and compare information at many different scales [13].

Some experts consider the ZUI interface paradigm as a flexible and realistic successor to the traditional windowing GUI. But little effort is currently spent developing ZUIs, while there are ongoing efforts for developing GUIs.

\section{Semantic Zoom}

In contrast to ordinary graphical zoom, Semantic Zoom does not only change parameters of a graphical representation, but modifies the selection and structure of data to be displayed. Semantic Zoom is a form of details on demand that lets the user see different amounts of detail in a view by zooming in and out [3]. More precisely: 
- Geometric (standard) zooming: The view depends on the physical properties of what is being viewed.

- Semantic zooming: Different representations for different spatial scales. When zooming away, instead of seeing a scaled down version of an object, see a different representation. The representation shown depends on the meaning to be imparted.

\subsection{Additional Features}

The information recovery in QbZ system has some additional features, such as: visual representation, definition of detail levels and navigation on the graph scheme, storage of queries for later use, conversion of a query form visual to textual representation or vice versa, among others.

The main advantages obtained using a direct manipulation visual techniques, as QbZ are:

1. shortening of the distance between the user's mental model of reality and the representation of such reality proposed by the computer;

2. reduction of the dependency on the native language of the user;

3. ease in learning of the basic functionality of the interaction;

4. high efficiency rate obtained also by expert users, partly because of the possibility of defining new functions and features;

5. significant reduction in the error rate;

\section{Test Results}

In this section we will present a running example to illustrate the use of QbZ. Moreover, we will present and comment the results of an experimental usability test.

\subsection{Running Example}

In order to demonstrate the efficiency of the proposed approach a QbZ prototype was implemented, called NanoZoom, using NanoBase as DBMS. NanoBase provides a relational view for access to data on the JME CLCD/MIDP platform, allowing the use of DDL/DML clauses, integrity restrictions, as well as several index structures [10]. NanoZoom was implemented using the JME CLDC/MIDP platform.

\subsubsection{Example 1}

Consider the following task: for each dependent retrieve your ID, your name and the name of the employee involved. Figure 4 shows the QbZ Expression, generated by the QbZ external model, to retrieve the requested data. Figure 5 shows the QbZ operator graph, generated by the QbZ internal model, for the Example 1. 


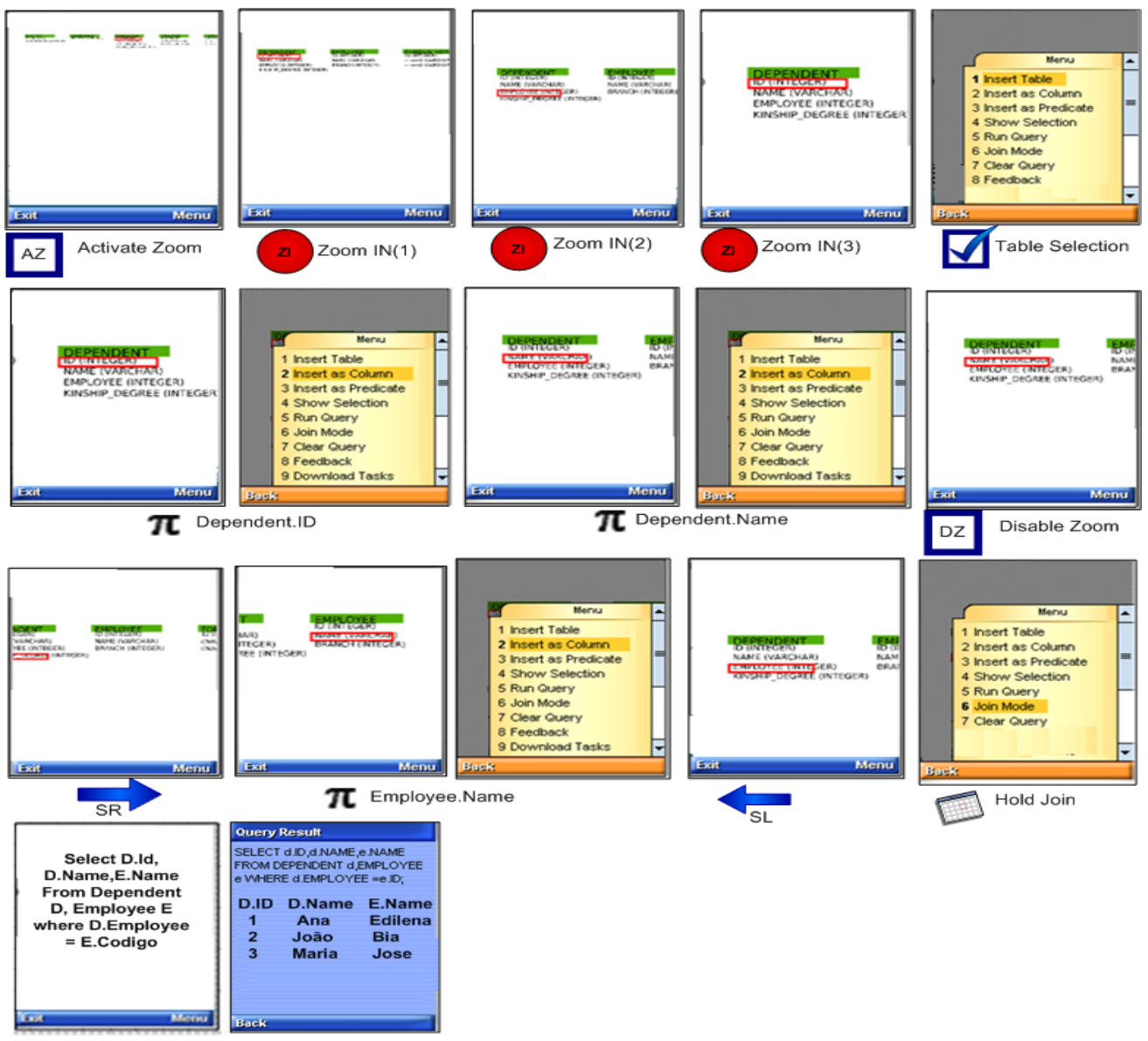

Figure 4: QbZ Expression for the Example 1.

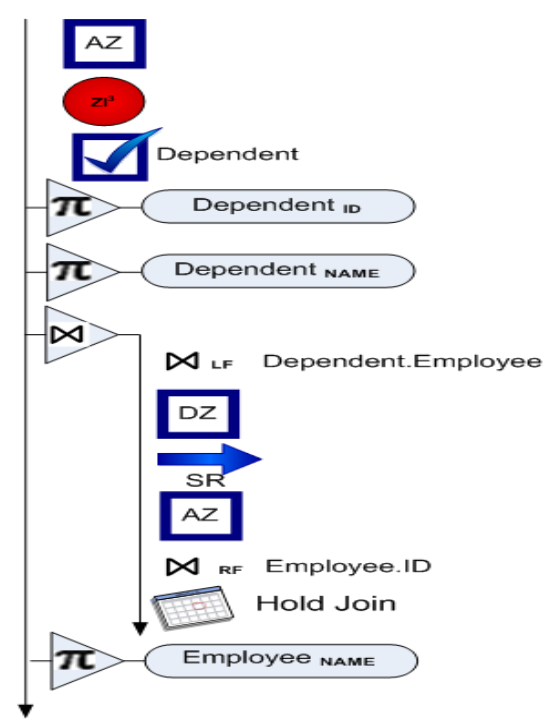

Figure 5: QbZ Operator Graph for the Example 1. 
This QbZ graph is translated to a relational algebra expression:

$\Pi_{\text {D.Id,D.Name,E.Name }}\left(\right.$ Dependent $\bowtie_{\text {D.Employee }=\text { E.Id }}$ Employee $)$

Next, the relational algebra expression is translated to a SQL clause:

SELECT D.ID, D.NAME, E.NAME

FROM DEPENDENT D, EMPLOYEE E

WHRE D.EMPLOYEE $=$ E.ID

Finally, the SQL expression is send to the DBMS and the result is displayed.

\section{$5.2 \quad$ Usability Testing Results}

Usability testing is a technique used to evaluate a product by testing it on users. This can be seen as an irreplaceable usability practice, since it gives direct input on how real users use the system. Usability testing focuses on measuring a human-made product's capacity to meet its intended purpose. In order o evaluate QbZ usability we used a methodology similar to Hallway testing (or Hall Intercept Testing), which is a general methodology of usability testing. Rather than using an in-house, trained group of testers, indicative of a cross-section of end users, are brought in to test the product. The name of the technique refers to the fact that the testers should be random people who pass by in the hallway. For this test we use a group of twenty computer science students, a database scheme with three relations (Employee, Dependent and Branch) and a task list with six questions. Figure 6 shows the students performance and their complexity perception.

\begin{tabular}{|c|c|c|c|c|c|c|}
\hline \multicolumn{7}{|c|}{ Task List } \\
\hline & \multicolumn{3}{|c|}{ Executed } & \multicolumn{3}{|c|}{ Difficulty Level } \\
\hline Task & Yes & No & Partial & Low & Medium & High \\
\hline 1. List all dependents & 20 & & & 20 & & \\
\hline 2. List id and name of all branches & 20 & & & 20 & & \\
\hline $\begin{array}{l}\text { 3. List id and name of the dependent with } \\
\text { id }=1\end{array}$ & 20 & & & 20 & & \\
\hline $\begin{array}{l}\text { 4. List id and name of all employees work- } \\
\text { ing in the branch with id }=1\end{array}$ & 20 & & & 20 & & \\
\hline $\begin{array}{l}\text { 5. List id and name of employees working } \\
\text { in branch with id }=1 \text { or id }=2\end{array}$ & 17 & 3 & & 16 & 1 & 3 \\
\hline $\begin{array}{l}\text { 6. List the name of each dependent with } \\
\text { the name of the employee responsible for } \\
\text { him }\end{array}$ & 8 & 12 & & 4 & 10 & 6 \\
\hline
\end{tabular}

Figure 6: Task Execution Results.

In Figure 6, we can observe that three users had difficulties in carrying out the task 5 . This fact was due to the difficulty of the user to select the OR operator, mainly because when he chooses to add a connective, the AND operator was selected by default, and the operator exchange was not intuitive. Therefore, there is a need to use a help option or a hint. Task 6 was the one with the highest difficulty degree. According to users reports, the action of associate relations using relationships does not have an easy learning. Thus, the relationships definitions (by foreign keys) need to be improved. 


\section{Conclusions And Future Works}

In this article we present QbZ (Query by Zoom), a query language based on Semantic Zoom, for portable devices. This language makes it easier to locate and access data stored in the mobile devices. The information recovery in QbZ system has yet some additional features, such as: visual representation, definition of detail levels and navigation on the graph scheme, storage of queries for later use, conversion of a query form visual to textual representation or vice versa, among others.

As future works we intend to use the TinySVG graphical library in the implementation of the visual interface and implement other relational operators like sort and aggregation. In addition, we intend to provide support for complex queries, such as those involving sub-queries, for example.

\section{References}

[1] R. Ahmad and S. Abdul-Kareem. A free-form database query language for mobile phones. Communications and Mobile Computing, International Conference on, 3:279-284, 2009.

[2] S. Bhalla and M. Hasegawa. A query interface for ubiqitous access to database resources. In COMAD '06: Proceedings of Conference on Management of Data, New Delhi, India, 2006.

[3] M. N. K. Boulos. The use of interactive graphical maps for browsing medical/health internet information resources. International Journal of Health Geographics, 2, 2003.

[4] T. Buering, J. Gerken, and H. Reiterer. User interaction with scatterplots on small screens - a comparative evaluation of geometric-semantic zoom and fisheye distortion. IEEE Transactions on Visualization and Computer Graphics, 12(5):829-836, 2006.

[5] T. Catarci, M. F. Costabile, S. Levialdi, and C. Batini. Visual query system for databases: A survey. Journal of Visual Languages and Computing, 1997.

[6] E. Chang, F. Seide, H. M. Meng, Z. Chen, Y. Shi, and Y.-C. Li. A system for spoken query information retrieval on mobile devices. Speech and Audio Processing, IEEE Transactions on Publication, 10:531- 541, 2002.

[7] Y.-H. Chang. A graphical query language for mobile information systems. SIGMOD Rec., $32(1): 20-25,2003$.

[8] B. Czejdo, R. Elmasri, M. Rusinkiewicz, and D. W. Embley. A graphical data manipulation language for an extended entity-relationship model. Computer, 23(3):26-36, 1990.

[9] E. Diep and R. J. K. Jacob. Visualizing e-mail with a semantically zoomable interface. In INFOVIS '04: Proceedings of the IEEE Symposium on Information Visualization, Washington, DC, USA, 2004. IEEE Computer Society.

[10] L. Eloy, V. Arajo, J. M. Monteiro, and A. Brayner. A tiny relation database manager for the jme cldc/midp platform. Revista Tecnologia, 29(1):7-15, 2008.

[11] HTC. Htc x9501. 2008. Available at http://www.htc.com/www/product.aspx.

[12] A. Massari and S. W. P. K. Chrysanthis. Supporting mobile database access through query by icons. In Distributed and Parallel Databases Jour, pages 4-249, 1996.

[13] K. Perlin and D. Fox. Pad: An alternative approach to the computer interface. In Proceedings of SIGGRAPH 93, 1993.

[14] S. Polyviou, G. Samaras, and P. Evripidou. A relationally complete visual query language for heterogeneous data sources and pervasive querying. In ICDE '05: Proceedings of the 21st International Conference on Data Engineering, pages 471-482, Washington, DC, USA, 2005. IEEE Computer Society. 